\title{
Radiological findings in acute Haemophilus influenzae pulmonary infection
}

\author{
F OKADA, MD, Y ANDO, MD, S TANOUE, MD, R ISHII, MD, S MATSUSHITA, MD, A ONO, MD, T MAEDA \\ and $\mathrm{H}$ MORI, MD
}

Oita University Faculty of Medicine, Yufu, Oita, Japan

\begin{abstract}
Objective: The aim of this study was to assess pulmonary thin-section CT findings in patients with acute Haemophilus influenzae pulmonary infection.

Methods: Thin-section CT scans obtained between January 2004 and March 2009 from 434 patients with acute $H$. influenzae pulmonary infection were retrospectively evaluated. Patients with concurrent infection diseases, including Streptococcus pneumoniae $(n=76)$, Staphylococcus aureus $(n=58)$ or multiple pathogens $(n=89)$ were excluded from this study. Thus, our study group comprised 211 patients (106 men, 105 women; age range, 16-91 years, mean, 63.9 years). Underlying diseases included cardiac disease $(n=35)$, pulmonary emphysema $(n=23)$, post-operative status for malignancy $(n=20)$ and bronchial asthma $(n=15)$. Frequencies of CT patterns and disease distribution of parenchymal abnormalities, lymph node enlargement and pleural effusion were assessed by thin-section $\mathrm{CT}$.

Results: The CT findings in patients with $H$. influenzae pulmonary infection consisted mainly of ground-glass opacity $(n=185)$, bronchial wall thickening $(n=181)$, centrilobular nodules $(n=137)$ and consolidation $(n=112)$. These abnormalities were predominantly seen in the peripheral lung parenchyma $(n=108)$. Pleural effusion was found in 22 patients. Two patients had mediastinal lymph node enlargement.

Conclusion: These findings in elderly patients with smoking habits or cardiac disease may be characteristic CT findings of $H$. influenzae pulmonary infection.
\end{abstract} Received 20 February 2010
Revised 6 May 2010 Accepted 14 May 2010

DOI: $10.1259 / \mathrm{bjr} / 48077494$

(C) 2012 The British Institute of Radiology
Haemophilus influenzae is an important pneumonia pathogen because of its severity, high incidence of complications and high mortality. This Gram-negative bacillus frequently colonises the human upper respiratory tract, especially the nasopharynx, and is considered to form part of the normal respiratory flora [1]. Most $H$. influenzae infections are the result of direct extension from the nasopharynx to the lower respiratory tract [1].

$H$. influenzae infection has received increasing attention because it is an important factor in the acute exacerbation of chronic obstructive pulmonary disease (COPD) [1, 2]. Acute exacerbation is a frequent event during the prolonged chronic course of COPD, which entails significant morbidity and mortality, and the main aetiology for the majority of episodes is infection.

The mortality rate in patients with $H$. influenzae pneumonia has been reported as 10-42\% [3-6]. Moreover, nosocomial outbreaks caused by $H$. influenzae have been reported [7]. Therefore, it is important to identify the risk factors associated with $H$. influenzae infection and to evaluate the radiological findings so that no time is lost in initiating appropriate management.

Several studies have presented the clinical and microbiological findings in patients with $H$. influenzae infection $[1-4,6,7]$. The characteristics of $H$. influenzae

\footnotetext{
Address correspondence to: Dr Fumito Okada, Oita University Faculty of Medicine, 1-1 ldaigaoka, Hasama-machi, Oita 879-5593, Japan. E-mail: fumitook@med.oita-u.
}

pneumonia on plain radiography have also been described previously [1, 8]. Recently, Nei et al [8] have described CT findings of Mycoplasma pneumoniae pneumonia and community-acquired pneumonia caused by other organisms, including 12 patients with $H$. influenzae pneumonia. The CT finding of bronchial wall thickening in patients with $H$. influenzae pneumonia was more common than in patients with Streptococcus pneumoniae or Klebsiella pneumoniae.

However, to the best of our knowledge, no other English-language studies of pulmonary CT findings in patients with acute $H$. influenzae pneumonia have been published. This study aimed to assess the clinical findings and pulmonary thin-section CT findings in patients with acute $H$. influenzae pneumonia.

\section{Methods and materials}

Our institutional review board approved this retrospective study and waived informed consent.

Based on the patient populations from four institutions, we retrospectively identified 434 patients with acute pulmonary infections due solely to $H$. influenzae who had undergone chest thin-section CT scans between January 2004 and March 2009. We excluded 76 patients with S. pneumoniae, 58 with Staphylococcus aureus, 33 with methicillin-resistant $S$. aureus (MRSA), 31 with Moraxella catarrhalis, 22 with Pseudomonas aeruginosa, 6 with $K$. pneumoniae and some with other pathogens who were 
diagnosed with concurrent infectious diseases by serological tests and clinical findings. Moreover, seven patients with acute $H$. influenzae pulmonary infection were excluded because of poor image quality caused by motion artefacts, inadequate window level settings or for whom hard copies of the CT film had been destroyed. Thus, the study group comprised 211 patients (106 men, 105 women; age range, 16-91 years, mean, 63.9 years) with acute $H$. influenzae pulmonary infection.

The diagnosis was established by isolation of $H$. influenzae from sputum in 170 patients, sputum from the trachea in 24 patients and bronchoalveolar lavage fluid in 17. In 90 patients at 1 of our institutions, biotypes were examined, which consisted of Type I $(n=3)$, Type II $(n=41)$, Type III $(n=44)$, Type IV $(n=0)$, Type V $(n=1)$, Type VI $(n=1)$ and Type VII $(n=0)$. A patient was considered to have community-acquired pneumonia if, at the time of hospital admission, he/she presented with cough, with or without sputum, fever, leukocytosis or leukopenia, and had pulmonary infiltrates on chest radiographs. Among the 211 patients, 102 had community-acquired and 109 had nosocomial infections.

Patients with frequent various underlying disease, alcoholics and smokers were evaluated. For the purposes of this study, an alcoholic was defined as an individual with a daily consumption of $\geqslant 80 \mathrm{~g}$ of alcohol during the past 2 years [9], and a patient was considered to be a heavy smoker if he/she had smoked for more than 10 pack-years.

\section{CT examinations}

Thin-section CT examinations were performed with a variety of scanners, with $1-\mathrm{mm}$ collimation $(n=19)$ at $10 \mathrm{~mm}$ intervals from the apex of the lung to the diaphragm, or volumetrically with a multidetector CT scanner $(n=192)$ with $1-\mathrm{mm}$ reconstruction. The scans were obtained with the patient in the supine position at full inspiration and were reconstructed using a high spatial frequency algorithm.

Images were captured at window settings that allowed viewing of the lung parenchyma (window level, -600 to $-700 \mathrm{HU}$; window width, 1200-1500 HU) and the mediastinum (window level, 20-40 HU; window width, $400 \mathrm{HU})$.

The pulmonary CT scan was performed within 1-6 days (mean, 4.8 days) after the onset of respiratory symptoms. Intravenously administered contrast material was used in 10 patients.

\section{CT image interpretation}

Two chest radiologists (with 21 and 13 years of experience in chest CT image interpretation), who were aware of the underlying diagnoses, retrospectively and independently interpreted the CT scans. Conclusions were reached by consensus. On average, 2 sessions per week were reserved to review the CT scans, with a total of approximately 50 sessions.

CT images were assessed for the following radiological features: ground-glass opacity, consolidation, nodule, centrilobular nodules, bronchial wall thickening, interlobular septal thickening, intralobular reticular opacity, bronchiectasis, enlarged hilar/mediastinal lymph node(s) $(>1 \mathrm{~cm}$ diameter short axis) and pleural effusion. Areas of ground-glass opacity were defined as hazy increases in opacity without obscured vascular markings [10, 11]. Areas of consolidation were defined as areas of increased opacity that obscured normal lung markings [10, 11]. Centrilobular nodules were defined as those present around the peripheral pulmonary arterial branches or 3-5 mm from the pleura, interlobular septa or pulmonary veins. Interlobular septal thickening was defined as abnormal widening of the interlobular septa [11]. Intralobular reticular opacity was considered present when interlacing line shadows were separated by a few millimetres [10,11]. In addition, the frequency of the combination of each CT finding was evaluated.

The distribution of parenchymal disease was also noted. We also assessed whether the abnormal findings were located unilaterally or bilaterally. If the main lesion was predominantly located in the inner third of the lung, the disease was classified as having a central distribution. On the other hand, if the lesion was predominantly located in the outer third of the lung, the disease was classified as having a peripheral distribution. If the lesions showed no predominant distribution, the disease was classified as having a random distribution. In addition, zonal predominance was classified as upper, lower or random. Upper lung zone predominance was defined as the presence of most abnormalities at a level above the tracheal carina, while lower zone predominance was defined as most abnormalities being below the upper zone. When abnormalities showed no definite zonal predominance, the lung disease was considered to have a random distribution.

Follow-up CT examinations were performed 4 days to 2 months after antibiotic therapy in 39 patients, and followup chest radiographs were performed 1 day to 2 months after antibiotic therapy in 128 patients. These follow-up CT images and radiographs were also assessed.

\section{Results}

\section{Patients' background}

The underlying conditions of all patients are summarised in Table 1. Overall, 43 patients were chronic smokers, 20 were alcoholics and 15 were both alcoholics and chronic smokers. 35 patients had concomitant cardiac disease $(16.6 \%)$. Patients with pulmonary emphysema $(n=23,10.9 \%)$, post-operative malignancy $(n=20$, $9.5 \%)$, bronchial asthma $(n=15,7.1 \%)$, diabetes mellitus $(n=9,4.3 \%)$ or liver disorders $(n=8,3.8 \%)$ were included in the study.

10 of 102 patients with community-acquired and 15 of 109 patients with nosocomial infections were treated with antibiotic therapy prior to CT examination. However, most of the patients in the present study, including the patients who received antibiotic therapy prior to CT examinations, showed rapid progression of their respiratory symptoms. The most common presenting symptoms were cough (190 patients, 90.0\%), sputum (166 patients, 78.7\%), fever (139 patients, 65.9\%) and dyspnoea (28 patients, $13.3 \%$ ). 
Table 1. Patient characteristics and underlying conditions

\begin{tabular}{lr}
\hline Sex, M/F & $n(\%)$ \\
\hline Smoking habit & $43(20.4)$ \\
Cardiac disease & $35(16.6)$ \\
Pulmonary emphysema & $23(10.9)$ \\
Alcoholic & $20(9.5)$ \\
Asthma & $15(7.1)$ \\
Diabetes mellitus & $9(4.3)$ \\
Liver disorder & $8(3.8)$ \\
Collagen disease & $5(2.4)$ \\
Malignancy & $20(9.5)$ \\
Lung cancer & $3(1.4)$ \\
Gastric cancer & $2(0.9)$ \\
Oesophageal cancer & $4(1.9)$ \\
Colon cancer & $2(0.9)$ \\
Laryngopharyngeal cancer & $2(0.9)$ \\
Presenting symptoms & \\
Productive cough & $184(87.2)$ \\
Non-productive cough & $24(11.4)$ \\
Fever & $139(65.9)$ \\
Dyspnoea & $28(13.3)$ \\
General weakness & $26(12.3)$ \\
\hline
\end{tabular}

\section{CT patterns}

Chest CT scans revealed abnormalities in all patients with $H$. influenzae pulmonary infection (Table 2). Among the 211 patients, ground-glass opacity $(n=185,87.7 \%)$ (Figures 1-4) was the most frequently observed abnormality, followed by bronchial wall thickening $(n=$ $181,85.8 \%$; Figures $1-4)$, centrilobular nodules $(n=137$, $64.9 \%$; Figures 1, 2 and 4), consolidation $(n=112,53.1 \%$; Figures 2 and 3$)$ and bronchiectasis $(n=34,16.1 \%$; Figure 3). Nodules $(n=30,14.2 \%)$, intralobular reticular opacity $(n=21,10.0 \%)$ and interlobular septal thickening $(n=21,10.0 \%)$ were also observed. The most frequently observed combination of abnormalities was groundglass opacity and bronchial wall thickening $(n=161$, $76.3 \%$; Figures 1-4), followed by ground-glass opacity and centrilobular nodules ( $n=135,64.0 \%$; Figures 1,2 and 4), and bronchial wall thickening and centrilobular nodules ( $n=133,63.0 \%$; Figures 1, 2 and 4$)$.

\section{Disease distribution}

Of the 211 patients with $H$. influenzae infection, abnormal findings were found bilaterally in 123 (58.3\%), unilaterally in $88(41.7 \%)$ and peripherally in 108 (51.2\%; Figures 1, 2,

Table 2. Thoracic CT findings in 211 patients

\begin{tabular}{lc}
\hline Findings & $n(\%)$ \\
\hline Ground-glass attenuation & $185(87.7)$ \\
Bronchial wall thickening & $181(85.8)$ \\
Centrilobular nodules & $137(64.9)$ \\
Consolidation & $112(53.1)$ \\
Bronchiectasis & $34(16.1)$ \\
Nodules & $30(14.2)$ \\
Intralobular reticular opacity & $21(10.0)$ \\
Interlobular septal thickening & $21(10.0)$ \\
Cavity & $0(0)$ \\
Pleural effusion & $22(10.4)$ \\
Lymph node enlargement & $2(0.9)$ \\
\hline
\end{tabular}

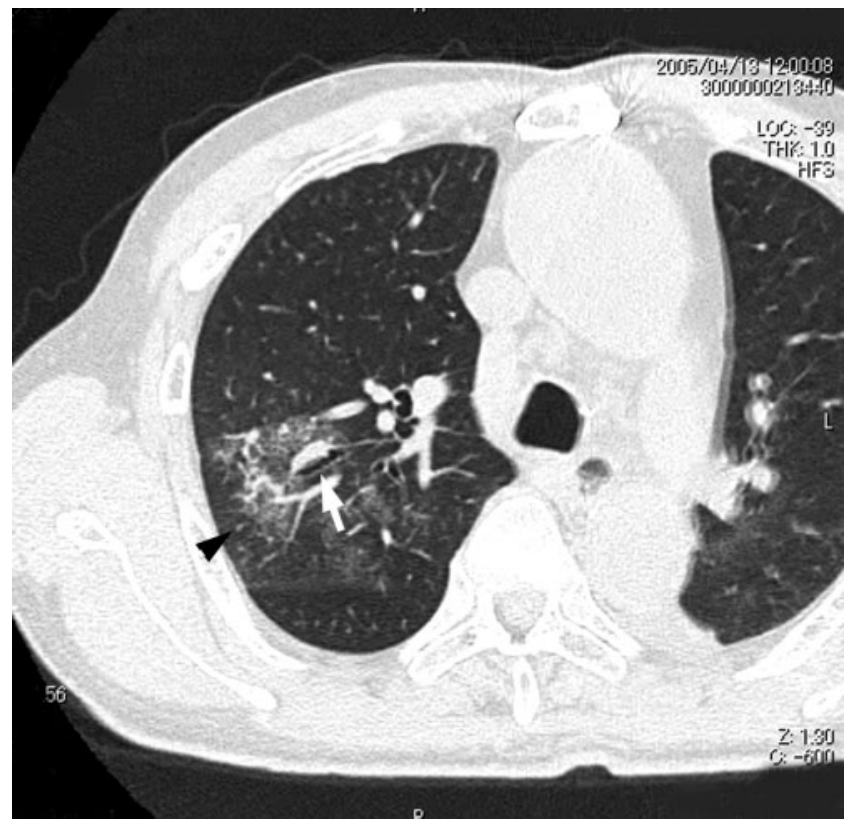

Figure 1. Acute Haemophilus influenzae infection in a 70year-old woman with cardiac disease at 4 days after onset of fever and cough. A transverse thin-section CT of the right upper lobe shows ground-glass opacity, bronchial wall thickening (arrow) and centrilobular nodules (arrowhead).

and 4). On the other hand, 103 patients showed a random distribution (48.8\%; Figure 3 ) and no patients had a predominantly central distribution. The predominant

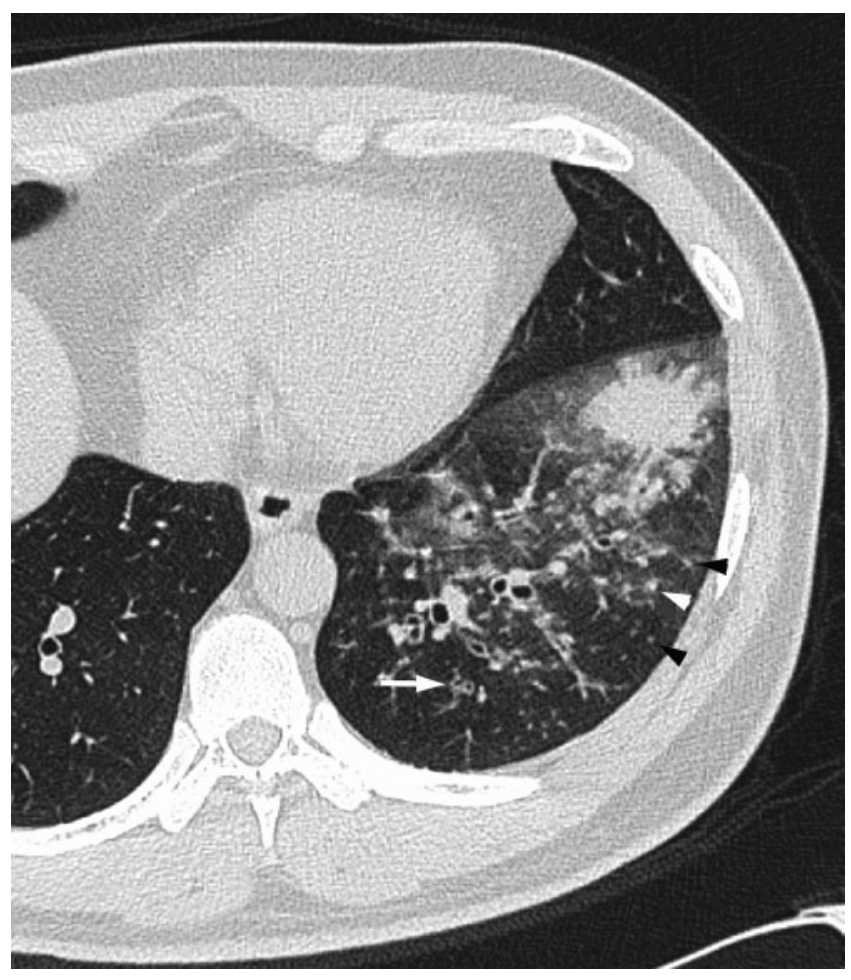

Figure 2. Acute Haemophilus influenzae infection in a 55year-old man with a smoking habit at 3 days after onset of fever and cough. A transverse thin-section CT of the left lower lobe shows consolidation, ground-glass opacity, bronchial wall thickening (arrow) and centrilobular nodules (arrowheads). 


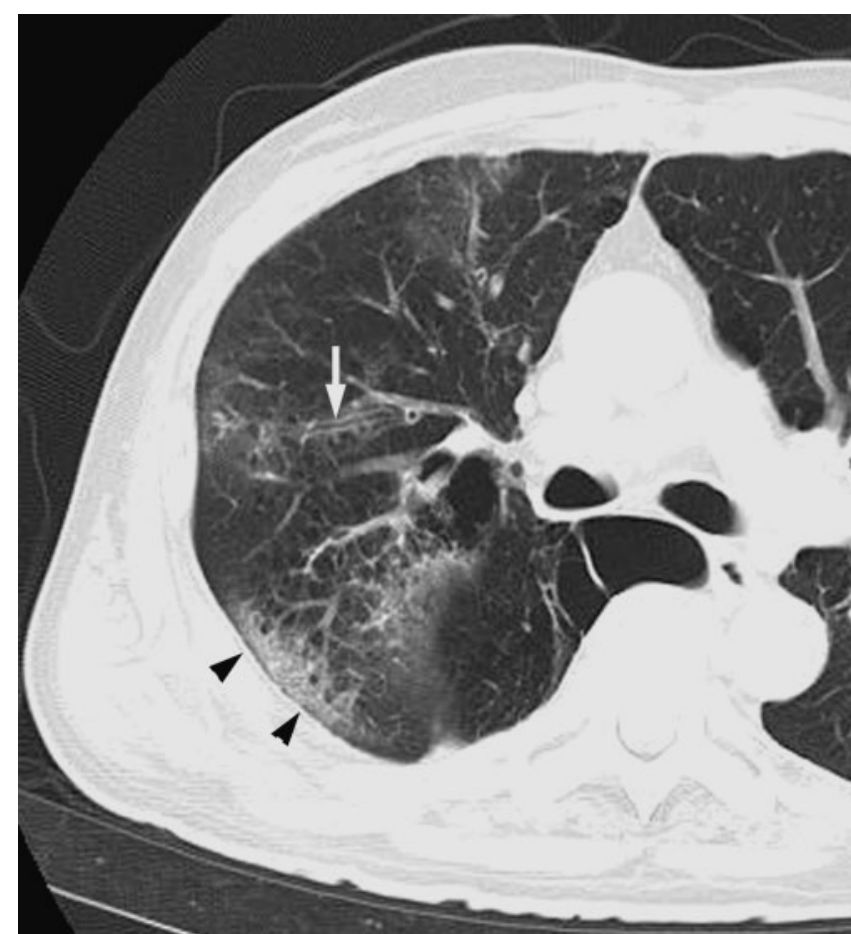

Figure 3. Acute Haemophilus influenzae infection in a 74year-old alcoholic man with pulmonary emphysema at 3 days after onset of fever, cough and dyspnoea. A transverse thinsection CT $2 \mathrm{~cm}$ below the tracheal carina shows ground-glass opacity, bronchial wall thickening (arrow) and consolidation (arrowheads).

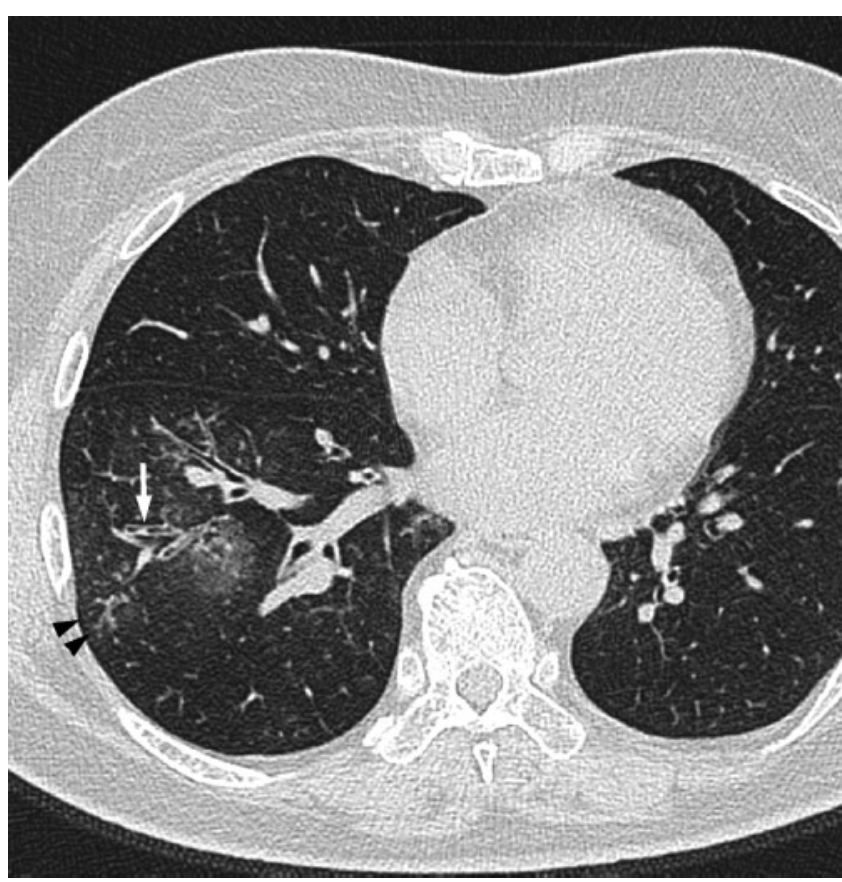

Figure 4. Acute Haemophilus influenzae infection in a 64year-old man at 3 days after the onset of cough with sputum. A transverse thin-section $\mathrm{CT}$ of the right lower lobe shows ground-glass opacity, bronchial wall thickening (arrow) and centrilobular nodules (arrowheads). zonal distribution of infection was the upper zone in 33 patients (15.6\%; Figure 1), the lower zone in 76 patients (36.0\%; Figures 2 and 4 ) and a random distribution in 102 patients (48.3\%; Figure 3$)$.

\section{Effusion and lymph nodes}

Bilateral pleural effusions were found in 5 patients $(2.4 \%)$, and unilateral pleural effusion was found in 17 patients (8.1\%) (Figure 2) with acute $H$. influenzae pulmonary infection. Two patients $(0.9 \%)$ had mediastinal lymph node enlargement.

\section{Follow-up study}

All 211 patients underwent antibiotic therapy. In 99 of 102 patients with community-acquired infections (97.1\%), the initial respiratory symptoms improved and the abnormal findings improved on follow-up CT examinations or chest radiographs. However, in the remaining three patients $(2.9 \%)$ with pulmonary emphysema and cardiac disease, abnormal findings such as ground-glass opacity and consolidation worsened on follow-up CT and these patients subsequently died. By comparison, in 101 of 109 patients with nosocomial infections (92.7\%), the initial respiratory symptoms improved and the abnormal findings improved on follow-up CT or radiographs. In the remaining eight patients $(7.3 \%)$, the abnormal parenchymal findings and pleural effusions worsened and the patients subsequently died. Of these patients, four had pulmonary emphysema, one had bronchial asthma and post-operative laryngeal cancer, one had bronchial asthma with cardiac disease, one was an alcoholic with diabetes mellitus and one had cardiac disease and diabetes mellitus.

\section{Discussion}

$H$. influenzae is one of the most clinically important Gram-negative bacterial pathogens, and is of great concern worldwide because infections can (1) exacerbate COPD, (2) cause pneumonia, particularly in older adults and (3) consist of a nosocomial respiratory tract pathogen $[1,12,13]$.

Exacerbation of COPD can be caused by many factors, including environmental irritants, heart failure or noncompliance with medication use [14]. However, most exacerbations are due to bacterial infection or viral infection [15]. Bacterial infection is a factor in $70-75 \%$ of exacerbations, with up to $60 \%$ caused by $H$. influenzae, $S$. pneumoniae or M. catarrhalis [2].

In addition, $H$. influenzae is the most common organism found in patients with acute exacerbation of chronic bronchitis and the second most common cause of community-acquired pneumonia in adults [16]; in addition, it is a nosocomial respiratory tract pathogen, mainly in elderly patients and those with underlying disease.

Underlying disease is found in 69-90\% of adults with $H$. influenzae disease [1, 4, 17-20]. Deulofeu et al [4] have reported that 30 of 43 patients $(69.8 \%)$ with $H$. influenzae disease had underlying conditions, including malignancy 
$(n=12,27.9 \%)$, alcoholism $(n=10,23.3 \%), \operatorname{COPD}(n=9$, $20.9 \%)$ and diabetes mellitus $(n=8,18.6 \%)$. Kofteridis et al [1] have reported that the underlying diseases in 45 patients with $H$. influenzae respiratory tract infection consisted mainly of COPD $(n=28,62.2 \%)$, followed by cardiovascular diseases $(n=16,35.6 \%)$, alcoholism $(n=16$, $35.6 \%)$ and diabetes mellitus $(n=13,28.9 \%)$. In our study, the underlying diseases in 211 patients were mainly a smoking habit $(n=43,20.4 \%)$, cardiac disease $(n=35$, $16.6 \%)$, pulmonary emphysema $(n=23,10.9 \%)$, alcoholism $(n=20,9.5 \%)$ and bronchial asthma $(n=15,7.1 \%)$. In the present study, the frequencies of underlying diseases were lower than those described in previous reports [1, 4, 17-20]. Furthermore, in the present study, the patients diagnosed with concurrent infectious diseases $(n=223,51.4 \%)$ were excluded. Therefore, the frequencies observed in this study might be lower than in previous reports.

In the present study, with $H$. influenzae alone, the average age was 63.9 years, which is similar to that found in previous reports $[1,4]$. S. pneumoniae, K. pneumoniae, M. pneumoniae and Chlamydia pneumoniae are also common pathogens involved in community-acquired or nosocomial pneumonia. The average age in patients with $H$. influenzae was similar to that in patients with $S$. pneumoniae, K. pneumoniae or C. pneumoniae pneumonia (60 years, 61.5 years and 57.7 years, respectively) [21-23]; however, the average age tended to be higher in patients with $H$. influenzae than in those with $M$. pneumoniae pneumonia (63.9 years vs 32-47.3 years, respectively) $[8,23,24]$.

Among the 211 patients in this study, 102 had community-acquired and 109 had nosocomial infections, which are similar findings to an earlier report [25] and suggest that $H$. influenzae is both a nosocomial respiratory pathogen and a community-acquired pathogen.

Regarding the presenting symptoms, all patients in the present study had several complaints such as fever, cough and sputum. There were no differences between patients with other pneumonias [22, 23].

In the present study, the mortality rate was $5.2 \%$ (11 of 211 patients), which was lower than that found in previous reports [3-6]. To the best of our knowledge, there are no studies in which additional pathogens were evaluated in patients with $H$. influenzae pneumonia. In the present study, in 223 of 434 patients (51.4\%) with acute $H$. influenzae pulmonary infection, one or more additional pathogens such as S. pneumoniae, M. catarrhalis, S. aureus or MRSA were found; the patients diagnosed with concurrent infectious diseases were excluded from this study. Moreover, in the present study, the frequencies of the underlying diseases were lower than those in previous reports. Therefore, the mortality rates in our patients with $H$. influenzae pulmonary infection might be lower than those in previous studies.

There are several case reports of patients with $H$. influenzae pulmonary infection [1,8]. However, few of these reports included chest radiographs.

Kofteridis et al [1] have reported that, in 34 of 45 patients with $H$. influenzae respiratory tract infection, pneumonia was observed on chest radiographs, and in 24 of the 34 patients with $H$. influenzae, segmental opacity on chest radiographs were characteristics that were similar to those reported previously. Nei et al [8] have studied whether CT findings of $M$. pneumoniae pneumonia could be distinguished from community-acquired pneumonia caused by other organisms, including 12 patients with $H$. influenzae pneumonia. The CT findings of bronchial wall thickening in patients with $M$. pneumoniae pneumonia was significantly more common than in patients with communityacquired pneumonia; however, the CT findings were more common in community-acquired pneumonia due to $H$. influenzae than with other pathogens (S. pneumoniae in 20 patients, K. pneumoniae in 2 and Legionella pneumophila in 1).

However, to the best of our knowledge, no other English-language studies of pulmonary CT findings in patients with acute $H$. influenzae pulmonary infection have been published.

We retrospectively evaluated the CT findings of 211 patients with acute $H$. influenzae pulmonary infection. The most common CT finding was ground-glass opacity followed by bronchial wall thickening, centrilobular nodules, consolidation and bronchiectasis. The abnormal findings were predominantly seen in the peripheral lungs.

Nambu et al [21] reported that the CT findings in 41 patients with $S$. pneumoniae pneumonia consisted mainly of consolidation, reticular opacity and centrilobular nodules $(90 \%, 39 \%$ and $32 \%$, respectively). We have previously reported chest CT findings in 198 patients with acute $K$. pneumoniae pneumonia alone [22], in 42 patients with M. pneumoniae pneumonia alone [23] and in 40 patients with $C$. pneumoniae pneumonia alone [23]. The frequency of bronchial wall thickening associated with $H$. influenzae infection was higher than that with $S$. pneumoniae, K. pneumoniae or C. pneumoniae infections $(85.8 \%$ vs $41 \%, 26.3 \%(p<0.001)$ and $35.0 \%(p<0.001)$, respectively) [21-23]. Moreover, the frequency of centrilobular nodules associated with $H$. influenzae infection was also higher than that with $S$. pneumoniae, $K$. pneumoniae or C. pneumoniae infections $(64.9 \%$ vs $32 \%$, $4.0 \%(p<0.001)$ and $7.5 \%(p<0.001)$, respectively) [21-23]. The frequencies of these features in patients with $H$. influenzae infection were similar to those seen in $M$. pneumoniae infection [23, 24]. However, M. pneumoniae is a well-known and common cause of atypical community-acquired pneumonia, mainly in young adults with no underlying diseases, and the average age of patients with $M$. pneumoniae infection was lower than that of patients with $H$. influenzae infection (32-36.6 years vs 63.9 years) $[8,24,26]$. The frequency of consolidation was lower than that with S. pneumoniae or K. pneumoniae (56.1\% vs 90 and $91.4 \%(p<0.001)$, respectively) [21, 22]. In addition, intralobular reticular opacity was less frequently seen with $H$. influenzae than with $K$. pneumoniae or C. pneumoniae infections $(10.0 \%$ vs $85.9 \%(p<0.001)$ and $70.0 \%(p<0.001)$, respectively) $[22,23]$.

Bilateral pleural effusion was seen in 5 patients (2.4\%), and unilateral pleural effusion was seen in 17 patients $(8.1 \%)$ with $H$. influenzae infection. The frequency of pleural effusions was lower than in patients with other pathogens such as S. pneumoniae, C. pneumoniae or $K$. pneumoniae (20\%, 25-30\% and 53\%, respectively) [21-23].

Two patients had mediastinal lymph node enlargement in our present study. The frequency of lymph node enlargement was also lower in these patients than in patients with S. pneumoniae, C. pneumoniae or 
M. pneumoniae $(0.9 \%$ vs $36 \%, 5-33 \%$ and $7.1-10 \%$, respectively) [21-24].

It should be noted that there are several limitations to our study. Firstly, this was a retrospective study and CT images were interpreted by consensus. Secondly, our study lacked a pathological correlation with specific CT findings such as consolidation and intralobular reticular opacity. Thirdly, the thin-section CT images were obtained at several institutions using different protocols. Fourthly, biotypes of $H$. influenzae were examined in only 90 patients at 1 of our institutions; moreover, serotype was not examined. However, no differences among each biotype were present on CT findings in 90 patients. As well, no differences on clinical findings were observed, which was similar to a previous report [4].

In summary, the thin-section CT manifestations in patients with $H$. influenzae pulmonary infection consisted mainly of ground-glass opacity, bronchial wall thickening and centrilobular nodules in the lung periphery, with a low frequency of pleural effusion or lymph node enlargement. These findings in elderly patients with a smoking habit, cardiac disease or pulmonary emphysema may be characteristic CT findings of $H$. influenzae pulmonary infection.

\section{References}

1. Kofteridis D, Samonis G, Mantadakis E, Maraki S, Chrysofakis G, Alegakis D, et al. Lower respiratory tract infections caused by Haemophilus influenzae: clinical features and predictors of outcome. Med Sci Monit 2009;15:135-9.

2. Soler N, Torres A, Ewing S, Gonzalez J, Celis R, El-Ebiary $\mathrm{M}$, et al. Bronchial microbial patterns in severe exacerbations of chronic obstructive pulmonary disease (COPD) requiring mechanical ventilation. Am J Respir Crit Care Med 1998;157:1498-505.

3. Farley MM, Stephens DS, Brachman PS Jr, Harvey RC, Smith JD, Wenger JD. Invasive Haemophilus influenzae in adults: a prospective, population-based surveillance. Ann Intern Med 1992;116:806-12.

4. Deulofeu F, Nava JM, Bella F, Marti C, Morera MA, Font B, et al. Prospective epidemiological study of invasive Haemophilus influenzae disease in adults. Eur J Clin Microbiol Infect Dis 1994;13:633-8.

5. Moreno S, Martinez R, Barros C, Gonzalez-Lahoz J, FarciaDelgado E, Bouza E. Latent Haemophilus influenzae pneumonia in patients with HIV. AIDS 1991;5:967-70.

6. Berk SL, Shirley A, Holtsclaw A, Wiener SL, Smith JK. Nontypeable Haemophilus influenzae in the elderly. Arch Intern Med 1982;142:537-9.

7. Van Dort M, Walden C, Walker ES, Reynolds SA, Levy F, Sarubi FA. An outbreak of infections caused by nontypeable Haemophilus influenzae in an extended care facility. J Hosp Infect 2007;66:59-64.

8. Nei T, Yamano Y, Sakai F, Kudoh S. Mycoplasma pneumoniae pneumonia: differential diagnosis by computerized tomography. Intern Med 2007;46:1083-7.

9. Torres A, Serra-Batlles J, Ferrer A, Jimenez P, Celis R, Cobo E, et al. Severe community-acquired pneumonia-epidemiology and prognostic factors. Am Rev Respir Dis 1991;144:312-18.

10. Webb WR, Muller NL, Naidich DP. High-resolution computed tomography findings of lung disease. In: Webb
WR, Muller NL, Naidich DP, eds. High-resolution CT of the lung. 3rd edn. Philadelphia, PA: Lippincott Williams \& Wilkins, 2001: 71-92.

11. Austin JH, Muller NL, Friedman PJ, Hansell DM, Naidlich DO, Remy-Jardin M, et al. Glossary of terms for CT of the lungs: recommendations of the Nomenclature Committee of the Fleischner Society. Radiology 1996;200:327-31.

12. Miller EH, Capla ES. Nosocomial Haemophilus pneumonia in patients with severe trauma. Surg Gynecol Obstet 1984; 159:153-6.

13. Simon HB, Southwick FS, Moellering RC Jr, Sherman E. Haemophilus influenzae in hospitalized adults: current perspectives. Am J Med 1980;69:219-26.

14. Voelkel NF, Tuder R. COPD: exacerbation. Chest 2000;117: S376-9.

15. Fein A, Fein AM. Management of acute exacerbations in chronic obstructive pulmonary disease. Curr Opin Pul Med 2006:6:122-6.

16. Moxon ER, Murphy FT. Haemophilus influenzae. In: Mandell GL, Douglas RG, Beet JE, eds. Principles and practice of infectious diseases. 6th edn. New York: Churchill Livingstone, 2005: 2369-78.

17. Trollfors B, Claesson B, Lagergård T, Sandberg T. Incidence, predisposing factors and manifestations of invasive Haemophilus influenzae infections in adults. Eur J Clin Microbiol 1984;3:180-4.

18. Granoff DM, Basden M. Haemophilus influenzae infections in Fresno Country, California: A prospective study of the effects of age, race, and contact with a case on incidence of disease. J Infect Dis 1980;141:40-6.

19. Takala AK, Eskola J, van Alphen L. Spectrum of invasive Haemophilus influenzae Type b disease in adults. Arch Intern Med 1990;150:2573-6.

20. Wallace RJ Jr, Musher DM, Septimus EJ, McGowan JE Jr, Quinones FJ, Wiss K, et al. Haemophilus influenzae infections in adults: Characterization of strains by serotypes, biotypes, and beta-lactamase production. J Infect Dis 1981;144:101-6.

21. Nambu A, Saito A, Araki T, Ozawa K, Hiejima Y, Akao M, et al. Chlamydia pneumoniae: comparison with findings of Mycoplasma pneumoniae and Streptococcus pneumoniae at thin-section CT. Radiology 2006;238:330-8.

22. Okada F, Ando Y, Honda K, Nakayama T, Kiyonaga M, Ono A, et al. Clinical and pulmonary thin-section CT findings in acute K. pneumoniae pneumonia. Eur Radiol 2009;19:809-15.

23. Okada F, Ando Y, Wakisaka M, Matsumoto S, Mori H. Chlamydia pneumoniae pneumonia and Mycoplasma pneumoniae pneumonia: Comparison of clinical findings and CT findings. J Comput Assist Tomogr 2005;29:626-32.

24. Reittner P, Müller NL, Heyneman L, Johkoh T, Park JS, Lee $\mathrm{KS}$, et al. Mycoplasma pneumoniae pneumonia: Radiographic and high-resolution CT features in 28 patients. AJR Am J Roentgenol 2000;174:37-41.

25. Morrissey I, Maher K, Williams L, Shackcloth J, Felmingham D, Reynolds R; BSAC Working Parties on Resistance Surveillance. Non-susceptibility trends among Haemophilus influenzae and Moraxella catarrhalis from communityacquired respiratory tract infections in the UK and Ireland, 1999-2007. J Antimicrob Chemother 2008;62:ii97-103.

26. Atkinson TP, Balish MF, Waites KB. Epidemiology, clinical manifestations, pathogenesis and laboratory detection of Mycoplasma pneumoniae infections [Review]. FEMS Microbiol Rev 2008;32:956-73. 